\title{
Mathematical Mystery in a Cultural Game
}

\author{
Mustafa Gök ${ }^{1, *}$ \\ ${ }^{1}$ Van Yuzuncu Yil University, Turkey \\ *Correspondence: Van Yuzuncu Yil University, Turkey. E-mail: mustafagok@yyu.edu.tr
}

Received: September 8, 2020

Accepted: October 29, $2020 \quad$ Online Published: December 10, 2020

doi:10.5430/wje.v10n6p64

URL: https://doi.org/10.5430/wje.v10n6p64

\begin{abstract}
This study described the mathematical depth in a mathematical activity carried out in a village in Turkey's Eastern Anatolia Region. This activity presented in the context of the game reflects a cultural situation of doing mathematics over time. In this context, it can identify as a study of ethnomathematics. Therefore, the cultural game was introduced first, and then the mathematical depth behind this game was uncovered in all its aspects. Finally, the mathematical relationship behind the game was analysed in terms of mathematics education. The case study, as one of the qualitative research methods, was used in the study. The participants of the study consist of 1 person who knows, transmits, and teaches the cultural game. The game process and semi-structured interview that constituted the research data were recorded with a camera and a voice recording device. Descriptive analysis was used in the analysis of the interview. Findings of the study suggest that the cultural game is played without considering its mathematical depth, but that there is a rich mathematical depth behind it. The results also indicate that such games offer an effective way for adults learning mathematics. On the other hand, the study revealed that there could be different ways of thinking between school mathematics and ethnomathematics. It is thought that synthesizing mathematics with games that include ethnomathematics has the potential to provide students at diverse levels with an excellent mathematical experience.
\end{abstract}

Keywords: ethnomathematics, mathematical depth, problem-solving, mathematics education

\section{Introduction}

\subsection{Introduction to the Problem}

There are many relationships between games and mathematics (Wells, 2012). One can encounter situations where these two phenomena are intertwined in most places in the life adventures of people. On the other hand, games are assumed to be more general because of the existence of games where there may be no mathematical phenomenon recorded with time and space in the historical process.

To this end, many researchers have documented the games played in different parts of the world during certain periods in their studies. Although these documents, which are presented from an anthropological and cultural perspective, have many mathematical dimensions (Bishop, 1991), studies revealing the mathematical connections in these games are limited. In this context, since the ethnomathematics program includes traditional games, it can be investigated in terms of understanding the mathematics of cultural practices and finding ways of thinking differently (Albanese, Adamuz-Povedano, \& Bracho-López, 2017). In many games, there are many mathematical facts reflected implicitly or explicitly. This led to the idea that games can be used as a teaching tool to spread mathematical knowledge today.

As a reflection of this fact, the teaching of mathematical concepts through games has become popular in universities in recent years. In this context, I opened the "Teaching Mathematics with Games" course. In this course, each student is to require to present a unique project for the teaching of mathematics by games. I felt there was mathematical depth in a cultural activity presented in one of these projects. After a brief study, I learned that the mathematical dimension beyond this activity is related to the Chinese Remainder Theorem (CRT). This knowledge was not far from me as a mathematics educator. However, the fact that this activity was long-lasting in a village, the villagers who played this game were the ones without any formal education, and the solution offered a different algorithm than I be used to making to me, have focused my attention on it. 


\subsection{The Chinese Remainder Theorem}

The first definition of CRT was coined by a Chinese mathematician, Sun Zhu (or Sunzi), in the 3rd century AD (van Tilborg, 2011). This definition is problem number 26 in the third chapter of the Mathematical Classic of Master Sun by Sun Zhu and its explanation (Dauben, 2007). These are given below.

Now there is an unknown number of things. If we count by threes, there is a remainder 2 ; if we count by fives, there is a remainder 3; if we count by sevens, there is a remainder 2 . Method: If we count by threes and there is a remainder 2, put down 140 . If we count by fives and there is a remainder 3 , put down 63 . If we count by sevens and there is a remainder 2, put down 30. Add them to obtain 233 and subtract 210 to get the answer (Dauben, 2007)...

It is narrated that by this method, the Chinese generals calculated the number of their own soldiers by organizing them in a particular order, only the remainder ones. This method, so few people know, was called the secret calculation in ancient China. In this way, it is to be aimed to prevent enemies from learning about the number of soldiers (Ding, Pei, \& Salomaa, 1996).

In this method, the concealment of information that would be assumed to be the origin point of cryptology emerged as the concealment of a number (particularly the number of soldiers). This method was generalized from the perspective of mathematicians on the one hand and transformed in the context of information-culture interaction on the other. A reflection of this method used about 1800 years ago in the context of information-culture interplay has emerged as an algorithm functioning in a mysterious game.

The knowledge introduced as a mysterious game in this article and referred to as CRT in the dusty pages of mathematics history reveals that mathematics is a human activity and product (Fried, 2001). This approach requires math educators the idea that a cultural perspective in a math activity is valuable (Fried, 2014). This exploration should not only be limited to the place where mathematical knowledge is first to produce but should also include the study of how mathematical knowledge emerged in a different culture in various regions. It is stated that mathematical knowledge can be monitored in several ways in a culture (Suherman, 2018), and one of these ways is games.

The attraction of mathematical games is related to the mathematical knowledge behind it. People with different levels of mathematics knowledge perceive these games in different ways and enjoy them to the extent they perceive them. While some admire the algorithm or system that always works in these games, others are interested in why the structure here works this way. For example, a game in the context of CRT can be an equation solving activity for a student, a different context of CRT for an academic, while math for an adult can be a way to do the math. In this way, an effective way for adults learning mathematics can be presented.

The purpose of the study is to introduce a cultural game and reveal the mathematical knowledge that exists behind it. In this direction, the answers to the following questions are sought in the study.

- How did the cultural game come about, and how is it played?

-What are the ways that lead to mathematical knowledge behind the cultural game?

- What does the cultural game promise in terms of mathematics education?

\section{Method}

\subsection{Design of the Study}

Since the aim of the study was to reveal the mathematical knowledge in a cultural game, the case study, one of the qualitative research methods, was used. In this direction, the perspectives and opinions of individuals in real-life conditions are examined using triangulation (Yin, 2011). It can be a single person, a program, a group, and an institution unit of analysis because it involves the in-depth examination of a phenomenon within a limited system (Merriam, 2013). On the other hand, the results obtained are limited to the cases examined; therefore, there is no generalization concern (Çepni, 2010). In this study, people who know, play, and teach a cultural game in a village in Eastern Anatolia were selected as the unit of analysis.

\subsection{Participant of the Study}

In the mentioned village, it was determined that there were 2 people, one is 55 years old, and the other is 75 years old, who knew, played, and taught the cultural game that has been played by many people since the past. However, the older person could not be included in the study due to health problems. Therefore, the data on the cultural game were obtained from one person who voluntarily participated in the study. This person's native language is Kurdish. He learned Turkish in the military at the age of 20 . However, he can speak Turkish at an intermediate level. During the 
interview, I asked questions to my student who presented the project to help me because he knows Kurdish. The participant who continues his life as a farmer has not received any formal training. The criterion that makes the unit of analysis specific here is that a person who has not received any training skilfully plays a game that is likely to have been a deep mathematical relationship for many years.

\subsection{Data Collection Tools and Analysis of Data}

The game process and semi-structured interview that constituted the research data were recorded with a camera and a voice recording device. In addition to the demographic questions, the participants were asked "How long has the game been played in this village?", "Who taught you this game?", "How is the game played?", "What is the secret algorithm in the game?", and "How did you get the algorithm in the game?".

The recording data obtained from the interview was first transferred to the digital environment by listening again. Then, it was arranged for analysis. Descriptive analysis was used in the analysis of the interview. Besides, the mathematical dimension of the cultural game obtained in the interview was revealed. This approach, which aims to reach the mathematical knowledge behind the game, can be specified as a priori analysis of the game. Besides, the outputs of the study also provide indications for at which grade levels a posteriori analysis can be carried out.

\subsection{Validity and Reliability of the Study}

Voluntary participation of the participants and the triangulation of the collected data in scientific studies increase the validity of the research (Merriam, 2013; Yıldırım \& Şimşek, 2016). In this study, the data were shared with the participants, and the accuracy of it was confirmed. Besides, it can be stated that data triangulation is ensured because different tools such as observation, interview, and voice recording are used in the data collection process. On the other hand, reliability is the degree to which the data obtained with the results show consistency (Merriam, 2013). Miles and Huberman (1994) explained that what meant consistently is the relative stability of the research results in terms of time, researcher, and space. In this study, it can be stated that reliability has increased since the process of reaching the cultural game, and the mathematical meaning of this process is clearly stated.

\section{Results}

This section consists of three subsections. The first one includes the introduction of the cultural game, the second is its mathematical analysis, and the third is about the reflection of the obtained results on mathematics education.

\subsection{Mystery in a Hidden Cultural Game}

Eastern Anatolia region of Turkey is famous for its long and cold winter nights. In this region, there are different games that are appropriately named besides known games such as chess and checkers. On winter nights, local people perform an activity which we cannot determine precisely how long it has been played, but which is rumoured to have been learned from Iran(Note 1). I think it would be a more accurate approach to describe it as a mathematical game, as they do it in the form of doing math, and they do it for the purpose of having fun on these long nights.

One of the two people(Note 2) who knew this game in Yeşilağaç village of Saray district in Van province was a 55 years old person named Rifat Gülçig who never went to school, and I reached some information about this person through my student who presented the project about the game. Since Kurdish is the mother tongue of this person (the one on the right in Figure 1) and the interview is conducted in Turkish, some of the sentences may be difficult to understand.

Student: Sir, how long has this game been played, how many years have you been playing it?

Rifat: I don't know, when I was born, folks were playing. Everyone is playing. I don't know how many years, maybe 100 years or 200 years.

Student: Who taught you?

Rifat: My grandfather, my father.

Student: Your father taught. So how is the solution?

Rifat: The solution is, well, 70 of how many numbers are remainder by three (remainder by 3 ).

Student: You multiply by 70 .

Rifat: (Used a word meaning yes in Kurdish) One remaining divided by five (remainder by 3) 21 .

Student: You multiply by 21 . 
Rifat: How many are left in seven 15.

Student: You multiply the remainder and seven by 15 . You subtract 100, how's that?

Rifat: For 100 (With regards to 100 numbers), well, for example, 120 (giving an example), you subtract 100. The remainder is 20 , and you subtract 5 , and the remainder is 15 .

Student: You always subtract 100s.

Rifat: You always do it. You subtract every 100s. 105, 210, or 315. (Means subtracting these numbers)

Student: (They are playing the game to show how the game is played). Okay, sir, let's tell the remainder three by three.

Novice Player: I got it. (He picked a random place on the prayer beads (tasbih) and started counting threes) remainder 1 from three.

Rifat: Okay. 70.

Novice Player: (Counting up to the same place by 5) Remainder 1 from five.

Rifat: Okay. 90, 91. What is the remainder by seven?

Novice Player: (counting up to the same place by 7) Remainder 4 from seven.

Rifat: Four, 60, 91, 151. Subtract the hundred as well. 51. Subtract 5, and it is 46.

Novice Player: (counting) 46. Successful solution.

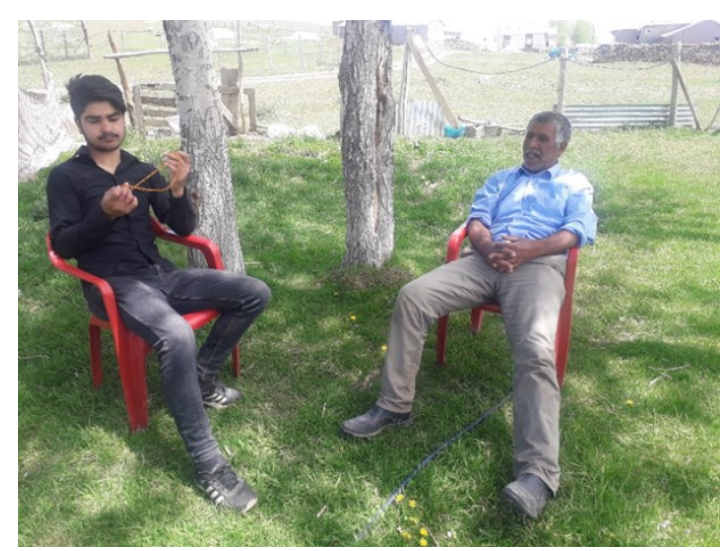

Figure 1. A moment from the Interview and Game Process

From the dialogue, it is understood that the cultural game has been played by many in the village for more than a century. When asked whether it knew where the factors (numbers $70,21,15$ ) used in the algorithm for the solution came from, they explained that they did not know this but should be used in this way. According to the information the researcher received from the student who presented the report on the cultural game, it was determined that the participant saw this game as a way of doing mathematics or calculating.

This game can be likened to games characterized as "think of a number." In a game where two people play each other, one of the players determines a place in the prayer beads and tells the opponent the remainder of that number by 3,5 , and 7 . Based on this information, the opponent is asked to estimate the target number. What is interesting at this point is the depth in one of those (villagers) and solution approaches that carry out this activity. However, it is also interesting to designate the place of the determined bead with the prayer bead. There is also the use of the prayer bead as a means of solution, albeit rare. This approach is discussed in the next section.

Although the prayer beads are often used as a material for finding the target number in the game, the game is also played with small stones. I assume that the prayer beads are used because it is the most suitable material for this game. The prayer beads consist of a rope, one imam, 99 beads, and two dividers. (Figure 2) The rope is connected to 33 beads. Then a divider is attached at each end of the rope. Then again, 33 beads are connected through both ends. Finally, the ends of the rope are joined together in an imam, and the prayer beads are created. The prayer beads, which is found in almost everyone in that region and used to perform some of the religious rituals, turns into a game 
material on winter evenings.

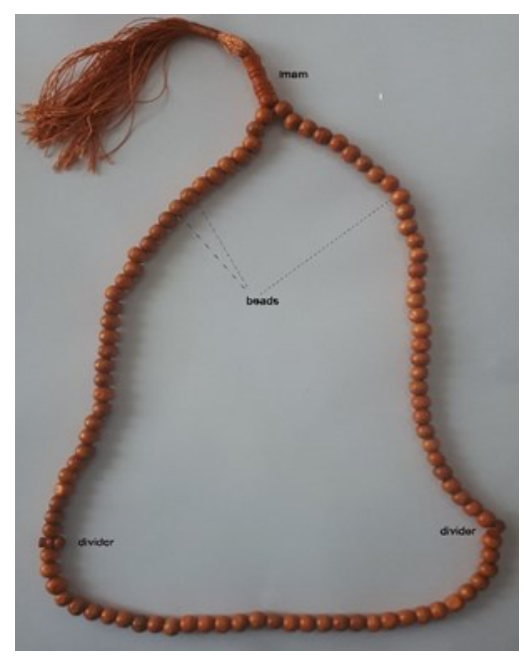

Figure 2. Prayer Beads (tasbih)

It is possible to suggest several different solution methods for finding the targeted number in this game. These are the origin of arithmetic (trial-and-error or algorithmic or mix) and algebraic.

\subsection{Mathematical Analysis of the Cultural Game}

Four different ways can be suggested to find the mathematical knowledge behind the cultural game. Each way was presented as a solution strategy.

\subsubsection{Solution with Trial-and-Error Strategy}

This solution approach in the game is often encountered in games between an older person and young people. This solution approach is based on sequential counting. In the game, the older player chooses one of the natural numbers from 1 to 104 and tells the younger opponent in turn what is the remainder of this number by the numbers 3,5 , and 7 (or the random ones that are remainder before he has determined the number). The other player tries to determine this number by trial and error method by using prayer beads. Suppose, for example, that the elderly player picks 57 . By hiding the number he holds, the old player will tell the younger player that when the number 57 (target number) is divided by 3,5 , and 7 , then 0,2 , and 1 are respectively the remainders. If the younger player is a novice, he will go in one direction on the prayer beads, starting with the 1st bead with his right hand, counting seven by seven, and starting with the 2 nd bead with his left hand, counting five each (It is wise to choose the appropriate one out of the three combinations here). After determining the intersection bead of these numbers, he will put a mark on this bead and check whether it is reached when counted three each from the beginning. If reached, the correct number is found. If the marked bead remains missing or overt when it is grouped into threes, it is understood that the marked bead is not the correct answer, and the game continues (See Table 1). In this context, it is attempted to find a second intersection by counting seven each and five each starting from the marked bead. In a shorter time, an addition of 35 of the first intersection point leads to the same solution path. Similarly, the beads up to this second intersection should be checked by counting them three each. In this way, the number that the elderly player thinks of can be determined experimentally in the game. These processes are given numerically in Table 1 and visually in Figure 3.

Table 1. A Sample Game Process

\begin{tabular}{lll}
\hline Consecutive Counting & Intersection 1 & Intersection 2 (Target Number) \\
\hline Seven each (right hand) & $1,8,15,22$ & $29,36,43,50,57$ \\
Five each (left hand) & $2,7,12,17,22$ & $27,32,37,42,47,52,57$ \\
Three each (control) & $3,6,9,12,15,18,21,24$ & $27,30,33,36,39,42,45,48,51,54,57$ \\
\hline
\end{tabular}

This process can continue with different combinations. For example, in one direction, the intersections obtained from the beginning by counting three and 2 nd bead from the beginning by counting five can be controlled by starting from the 1 st bead from the beginning by counting seven. However, I think that is enough for this solution. 


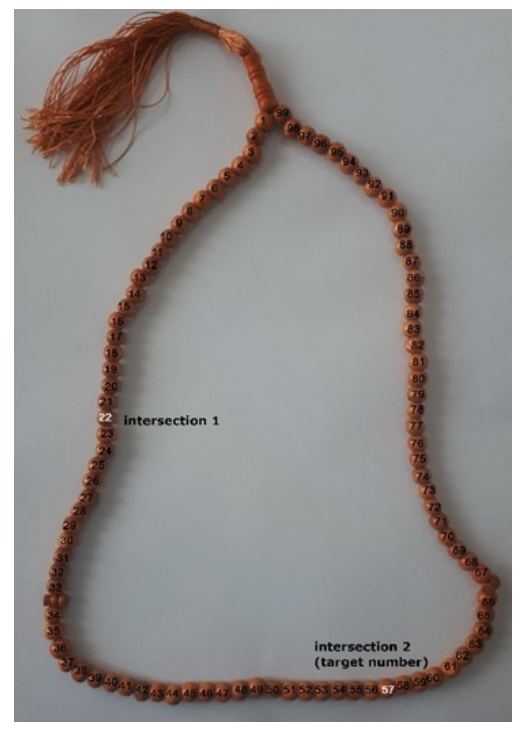

Figure 3. An Example Review

Finally, the use of the prayer beads in the solution approach of the trial-and-error strategy creates difficulty in some cases. In the prayer beads, only the target number is investigated through the beads. So dividers and imams are not calculated in this process (See Figure 3). In this case, the results between 0 and 100 on the prayer beads can be found by the novice players. However, larger numbers create problems. This situation indicates that there are situations novice players cannot work out through consecutive counting. In this context, when the numbers between 100 and 104 are selected in the game process, novice players cannot find the solution with the prayer beads.

\subsubsection{Algebraic Solution}

This solution approach is a method that individuals with a certain level of mathematics knowledge can use. This is because such problem situations are taught in the school curriculum and are generally solved by the method given below. The algebraic solution over the example given in the previous part is to be an integer $\mathrm{x}$ and $1 \leq \mathrm{x} \leq 104$,

$$
\begin{aligned}
& x \equiv 0(\bmod 3) \\
& x \equiv 2(\bmod 5) \\
& x \equiv 1(\bmod 7)
\end{aligned}
$$

and it is the common solution of the system consisting of congruence equations. As a matter of fact, according to the CRT, this system has only one solution under certain conditions (such as the numbers 3,5 , and 7 being relatively prime in pairs) (Shapiro, 1983). However, this solution is probably unknown to the villagers due to its being algebraic and containing a certain degree of knowledge of mathematics. In this sense, it is natural for arithmetic solutions to emerge.

\subsubsection{Solution with Mixed Methods}

Individuals who can make sense of the relationships between mathematical concepts can suggest the mixed approach that follows. This method is based on basic mathematical concepts (basic rules of division, linear equations in slope-intersection form, and arithmetic progress) (Firozzaman \& Firoz, 2017), and it is detailed below.

Let $A, p_{i}, q_{i}, r_{i}, i, j \in N$ and $A$ be divided by $p_{i}$ and the quotient is $q_{i}$ and the remainder is $r_{i}$. As per the basic rule of division,

$$
A=p_{i} \cdot q_{i}+\mathrm{r}_{\mathrm{i}}
$$

When this model is restricted to $\mathrm{n}$,

$$
q_{1}:=q_{1}^{(1)}=x ; q_{i+1}:=y_{i}=p_{i} \cdot q_{i} / p_{i+1}+\left(r_{i}-r_{i+1}\right) / p_{i+1}, i=1,2,3, \ldots, n
$$

We have an $n-1$ linear equation for the solution. The recurrence system provides an infinite number of solutions for $\mathrm{A}_{\mathrm{i}}^{(\mathrm{j})}$ with the smallest unique solution and unique solution set. The system produces the smallest unique value of $\mathrm{A}$, the first integer solution for divisions. The following arithmetic progress model is used for inputs in equation (2) to 
find other values of A.

$$
\begin{gathered}
q_{I}^{(j)}=q_{i}+L C M\left(\left\{p_{i}\right\}\right) \cdot(j-1) / p_{k}, i=k, i=1,2,3, \ldots, j=1,2,3, \ldots \\
A_{i}^{(j)}=p_{i} \cdot q_{i}^{(j)}+\mathrm{r}_{\mathrm{i}} \text { for any } \mathrm{i},
\end{gathered}
$$

Where $\mathrm{q}_{\mathrm{i}}^{(\mathrm{j})}$ is the $j$ th element of $i$ th quotient. The solution of the example given in the previous sections without breaking from generality with the mixed method is as follows. Assume that the integer we are looking for is A, then through the remainder rules of the division,

$$
A=3 \cdot q_{1}=5 \cdot q_{2}+2=7 \cdot q_{3}+1
$$

Here, the recurrence relation between the sections is as follows:

$$
q_{2}=3 \cdot q_{1} / 5+(0-2) / 5 ; q_{3}=5 \cdot q_{2} / 7+(2-1) / 7
$$

We can find an integer solution of $y$ from equation $3 x-2=5 k,(k \in Z)$. When $k=5$, the solution is $x=19, y=5$. Here, it should be investigated whether $\mathrm{z}:=\mathrm{q}_{3}$ is an integer. Indeed, $\mathrm{q}_{3}=8$. Therefore, one of the triple solutions given below for integer inputs and outputs is $\{(19,5,8)\}$. For each solution in the triple solution, only one solution is needed to find the set of input solutions. Others can be achieved through the use of arithmetic progression.

$$
q_{1}^{(j)}=q_{1}+\operatorname{LCM}(3,5,7) \cdot(j-1) / 3=19+35 \cdot(j-1), j \in N
$$

Here $q_{I}(j)=\{19,54,89, \ldots\}$ and if they are written in equation $(6)$, the solution set of the problem is $\mathrm{A}=\left\{\mathrm{A}^{(\mathrm{j})}\right\}=\{57$, $162,267, \ldots\}$. Therefore, the solution is 57 in the bounded set.

\subsubsection{Algorithmic Solution}

What makes this game meaningful is the fact that older and experienced players get to the target number in the game very quickly. For novice players, this situation is interesting and intriguing. At the end of a few games, the older player explains that he uses an algorithm to find the target number for his novice opponent. Mathematically, this algorithm can be expressed as follows. If the remainders of the selected number with the division of 3, 5, and 7 are a, $\mathrm{b}$, and $\mathrm{c}$, it can be presented as

$$
x \equiv 70 \cdot a+21 \cdot b+15 \cdot c(\bmod 105)
$$

If the algorithm is to be executed over the same example again, it will be as follows:

$$
\begin{aligned}
& x \equiv 70 \cdot 0+21 \cdot 2+15 \cdot 1(\bmod 105) \\
& x \equiv 57
\end{aligned}
$$

In other words, the remainder of the division of the target number by 3 is multiplied by 70 , the division of the target number by 5 is multiplied by 21 , and the division of the target number by 7 is multiplied by 15 . Assume that the number $\mathrm{x}$ is obtained after the results acquired are added. If the number $\mathrm{x}$ is between 0 and 105 , the number selected is $\mathrm{x}$, if between 105 and 210 , it is $\mathrm{x}-105$, and if between 210 and 315 , it is $\mathrm{x}-210$. It is significant to note that subtracting the multiples of 105 originates in the least common multiples of the numbers 3,5 , and 7 . The greatest value that $\mathrm{x}$ will get is $2 \cdot 70+4 \cdot 21+6 \cdot 15=314$. Subtracting 210 is sufficient to determine the number selected here. Accordingly, the number chosen is $314-210=104$.

The algorithm and mathematical dimension behind the game, which is defined as a way of doing math according to experienced players, is played regardless of what it is based on. They only perceive it as a rule that always works. It is not known why the remainder of the division of $x$ by three is multiplied by 70 instead of 35 , especially by the local people who use the algorithm. When asked about this situation, experienced players stated that they did not know why they multiplied by two but that they reached the correct answers in this way and that this should be here. The mathematical information beyond the algorithm that works here is a different interpretation of the CRT (Katz, 2009).

Assume that the division of number $x$ is respectively the remainders of $r_{1}, r_{2}, r_{3}, \ldots, r_{n}$ by the dividers of natural numbers of $\mathrm{m}_{1}, \mathrm{~m}_{2}, \mathrm{~m}_{3}, \ldots, \mathrm{m}_{\mathrm{n}}$ each of which is relatively prime in pairs. Therefore, the congruence system has a unique solution in modulo $\mathrm{M}=\mathrm{m}_{1} \cdot \mathrm{m}_{2} \cdot \mathrm{m}_{3} \cdot \ldots \cdot \mathrm{m}_{\mathrm{n}}$ (Shapiro, 1983).

$$
\begin{aligned}
& x \equiv r_{1} \quad\left(\bmod m_{1}\right) \\
& x \equiv r_{2} \quad\left(\bmod m_{2}\right) \\
& x \equiv r_{n} \quad\left(\bmod m_{n}\right)
\end{aligned}
$$

To generalize this algorithm, $\mathrm{M}_{\mathrm{i}}=\mathrm{M} / \mathrm{m}_{\mathrm{i}}$. Since $\mathrm{M}_{\mathrm{i}}$ and $\mathrm{m}_{\mathrm{i}}$ are relatively prime, there is only one $\mathrm{s}_{\mathrm{i}}$ so that $\mathrm{M}_{\mathrm{i}} \cdot \mathrm{s}_{\mathrm{i}} \equiv 1$ $\left(\bmod \mathrm{m}_{\mathrm{i}}\right)$. So, since this $\mathrm{s}_{\mathrm{i}}$ is modulo $\mathrm{m}_{\mathrm{i}}$, it is an element of the set $\left\{0,1, \ldots, \mathrm{m}_{\mathrm{i}}-1\right\}$. Accordingly, the algorithm has a unique solution according to the $\mathrm{M}$ modulo in the form of 


$$
\mathrm{x}=r_{i} \cdot \mathrm{M}_{\mathrm{i}} \cdot s_{i} \text { for } i=1,2,3, \ldots, n
$$

This general method was first introduced by Qin Jiushao in his Mathematical Treatise in Nine Sections (Katz, 2009).

\subsection{Reflections from the Cultural Game to Mathematics Education}

It is a known fact that models put forward regarding a problem or a situation in the development process of mathematics can explain many cases in mathematics. For example, the Fibonacci sequence, supposed to originate with the famous "rabbit problem", describes many problem situations (Vorobiev, 2002). Similarly, CRT is a general explanation of many problem situations, although the first examples are encountered in ancient China. In this study, CRT emerged in a cultural context as a way of doing math for adults. It is inevitable that this important theorem will be taught by transforming scientific knowledge in different ways at different levels for instructional purposes.

In school mathematics, in a certain range of the set of natural numbers, the CRT is used as problems related to finding a number whose remainder is known from its division by a series of numbers (Ministry of National Education [MoNE], 2018). It is observed that algebraic approaches are dominant to solve such problems in school mathematics. In this study, it is shown not only how these problems can be presented through a game that is a more innovative approach, but also how to give alternative solutions to the problems. The study, on the other hand, contains an engaging story for getting started with CRT in undergraduate math classes. For example, such a situation can be used when introducing the CRT subject in the undergraduate algebra course. Different solutions to this situation can be searched.

\section{Discussion}

Traces of mathematical relationships have been observed in most cultural games, such as mathematics, which is a human activity. Zaslavsky $(1998,2003)$ revealed a set of mathematical relationships that exist in many cultural games around the world. Similarly, Ascher (2001) examined how mathematical relationships in various activities related to ethnomathematics in Mongol culture can be used for instructional purposes. In the study in which one of these cultural games was presented, we witnessed that the villagers were enchanted by mathematics and that they did it in their own way as a way of doing mathematics for more than a century. This approach is reminiscent of adult mathematics learning, where villagers developed an understanding of the mathematics they need in daily life (Coben \& O'Donoghue, 2014; Wedege, 2010). This situation supports the claim that mathematics education should be considered from a broader scale perspective rather than limited to formal institutions (Knijnik \& Wanderer, 2010).

One of the significant results of this study is that games offer an effective way for adult mathematics learning. It is understood that this approach contributes to the regulars of the game in making simple mathematical calculations in their daily lives. On the other hand, the use of traditional tools in the transfer of knowledge shows that knowledge is carried to a cultural dimension. Here a game context is used where an algorithm based on CRT is the solution.

It is also significant that the problem and solution in the cultural game are similar to the problem and solution method given by Sun Zhu in the 3rd century AD. All we know about this is that the grandfather of my student who presented the project learned this game from an Iranian person. Naturally, the solution approaches of the villagers are of arithmetic origin. Although the algebraic method is dominant in school mathematics, arithmetic solutions of which use villagers indicate that different mathematics is formed. In a cultural study, Knijnik (2007) stated that the living conditions imposed on the villagers could lead to assigning a different meaning to mathematics. However, suggesting that this interesting method of solution, which works every time, and the relationships used in this method are coincidental is out of the question even for those who do not have a mathematical background.

This game has transferred to pass down on generations, and regulars of the game do not care about the mathematical depth in the game. Moreover, they do not know why the algorithmic solution works. This situation indicates that mathematical knowledge is carried out as an activity that has been worked out from a superficial point of view and that the mathematical depth felt beyond the game is ignored (Sánchez-Jiménez et al., 2020). It should not only protect the cultural values that we know to exist in a specific time and place, such as a cultural game or a puzzle, and which are about to disappear today. The mathematical relationships hidden in these games also need to be revealed in full depth. In this context, the mathematical depth of an arrangement related to discrete geometry in a puzzle in Mongolian culture can be examined (Ninjbat, 2020). Similarly, in this study, the mathematical depth of the activity within the scope of ethnomathematics, which has become a cultural game over time, has been revealed. Mathematical analysis of the cultural game and its reflections in school mathematics were effective in the emergence of another important result of the study. In this way, it was found that CRT is used in a very limited context in terms of mathematical knowledge in school mathematics. 
It can be argued that using a different method than the dominant approach in school mathematics in solving the cultural game and the presentation style of abstract mathematical knowledge activates a different mathematical way of thinking. As a matter of fact, it has been emphasized that different ways of thinking can emerge in such activities with mathematical depth (Albanese et al., 2017).

The relationship between traditional culture and mathematics is not limited to games. For example, many geometric relationships have been observed in traditional motifs (Risdiyanti, \& Prahmana, 2017; Suherman, 2018). It is stated that a cultural learning context supports mathematical problem-solving skills according to students' thinking levels (Nur, Waluya, Rochmad, \& Wardono, 2020). The integration of traditional games involving mathematical relationships (such as length, height, depth, symmetry) and teaching situations help students understand mathematical rules and concepts (Fouze, \& Amit, 2017). These results indicate that we should benefit more from the outputs of traditional culture in educational settings. Nevertheless, it can be said that we are facing an opposite trend.

It is among the natural tasks for a mathematician to reveal the mathematical dimension in a cultural game with mathematical depth. This study revealed the mathematical depth in a cultural game characterized as a "think of a number" game. I think that the mathematical depth in cultural games should be analysed, disseminated, and preserved as a mathematical heritage.

The presentation of mathematical knowledge without the story of its emergence is like giving it away from its soul. For example, the basic formulas for the product of Quaternions are given without mentioning Hamilton's story (Katz, 2009) engraving on a stone of the Brougham Bridge. On the other hand, the study emphasizes the importance of presenting the mathematical knowledge together with its story, as well as offering all possible solutions to the problem situation it has revealed.

\section{References}

Albanese, V., Adamuz-Povedano, N., \& Bracho-López, R. (2017). The evolution of ethnomathematics: Two theoretical views and two approaches to education. Ethnomathematics and its diverse approaches for mathematics education, 307-328. Springer, Cham. https://doi.org/10.1007/978-3-319-59220-6_13

Ascher, M. (2001). Learning with games of strategy from Mongolia. Teaching Children Mathematics, 8(2), 96-99.

Bishop, A. (1991). Mathematical enculturation: A cultural perspective on mathematics education. Dordrecht: Kluwer Academic Publishers.

Çepni, S. (2010). Araştırma ve proje çalışmalarına giriş̧ (5th ed.). Trabzon: Celepler Matbaacılık.

Coben, D., \& O’Donoghue, J. (2014). Adults learning mathematics. In S. Lerman (Ed.), Encyclopedia of mathematics education (pp. 15-23). Dordrecht: Springer. https://doi.org/10.1007/978-94-007-4978-8

Dauben, J. W. (2007). Chinese mathematics. In V. J. Katz (Ed.), The mathematics of Egypt, Mesopotamia, China, India and Islam, A sourcebook (pp. 187-384). Princeton, NJ: Princeton University Press.

Ding, C., Pei, D., \& Salomaa, A. (1996). Chinese remainder theorem: Applications in computing, coding, cryptography. River Edge, NJ: World Scientific Publishing.

Firozzaman, F., \& Firoz, F. (2017). Efficient remainder rule. International Journal of Mathematical Education in Science and Technology, 48(5), 756-762. https://doi.org/10.1080/0020739X.2016.1267807

Fouze, A. Q., \& Amit, M. (2017). Development of mathematical thinking through integration of ethnomathematic folklore game in math instruction. EURASIA Journal of Mathematics, Science and Technology Education, 14(2), 617-630. https://doi.org/10.12973/ejmste/80626

Fried, M. N. (2001). Can mathematics education and history of mathematics coexist? Science \& Education, 10, 391-408.

Fried, M. N. (2014). Mathematicians, historians of mathematics, mathematics teachers, and mathematics education researchers: The tense but ineluctable relations of four communities. In M. N. Fried \& T. Dreyfus (Eds.), Mathematics \& mathematics education: Searching for common ground (pp. 94-98). New York: Springer.

Katz, V. J. (2009). A history of mathematics. New York: Pearson.

Knijnik, G. (2007). Mathematics education and the Brazilian Landless Movement: three different mathematics in the context of the struggle for social justice. Philosophy of Mathematics Education Journal, 21(1), 1-18

Knijnik, G., \& Wanderer, F. (2010). Mathematics education and differential inclusion: A study about two Brazilian 
time-space forms of life. ZDM, 42(3-4), 349-360. https://doi.org/10.1007/s11858-010-0247-8

Merriam, S. B. (2013). Nitel vaka çalışması. In Çev: E. Karadağ. S. Turan (Ed.), Nitel araştırma desen ve uygulama için bir rehber içinde (3rd ed.). Ankara: Nobel.

Miles, M. B., \& Huberman A. M. (1994). Qualitative data analysis: An expanded sourcebook. Thousand Oaks, CA: Sage Publications.

Milli Eğitim Bakanlığı [Ministry of National Education] (MoNE). (2018). Ortaögretim matematik dersi (9, 10, 11 ve 12. siniflar) ögretim programı. Ankara: MEB Yayınları.

Ninjbat, U. (2020). "The Four Strongest" at the National Museum of Mongolia. The Mathematical Intelligencer, 42(2), 9-14. https://doi.org/10.1007/s00283-020-09979-9

Nur, A. S., Waluya, S. B., Rochmad, R., \& Wardono, W. (2020). Contextual learning with Ethnomathematics in enhancing the problem solving based on thinking levels. JRAMathEdu (Journal of Research and Advances in Mathematics Education), 5(3), 331-344. https://doi.org/10.23917/jramathedu.v5i3.11679

Risdiyanti, I., \& Prahmana, R. C. I. (2017). Ethnomathematics: Exploration in Javanese culture. Journal of Physics: Conference Series, 943(1), 012032. https://doi.org/10.1088/1742-6596/943/1/012032

Sánchez-Jiménez, E., Carrillo, D., Chevallard, Y., \& Bosch, M. (2020). The Second Spanish Republic and the project method: A view from the ATD. In M. Bosch, Y. Chevallard, F. J. García, \& J. Monaghan (Eds.), Working with the anthropological theory of the didactic in mathematics education: A comprehensive casebook (pp. 101-117). New York: Routledge.

Shapiro, H. N. (1983). Introduction to the theory of numbers, pure and applied mathematics. New York: Wiley-Interscience Publication, John Wiley \& Sons.

Suherman, S. (2018). Ethnomathematics: Eksploration of Traditional Crafts Tapis Lampung as Ilustration of Science, Technology, Engineering, and Mathematics (STEM). Eduma: Mathematics Education Learning and Teaching, 7(2), 21-30. https://doi.org/10.24235/eduma.v7i2.3085

Van Tilborg, H. C. A. (2011). Chinese remainder theorem. In H. C. A. van Tilborg \& S. Jajodia (Eds.), Encyclopedia of cryptography and security (pp. 201-201). Heidelberg: Springer.

Vorobiev, N. N. (2002). Fibonacci Numbers. Basel: Birkhäuser.

Wedege, T. (2010). The problem field of adults learning mathematics. In G. Griffiths \& D. Kaye (Eds.), Proceedings of 16th adults learning mathematics (pp. 13-24). London: Adults Learning Mathematics-A Research Forum.

Wells, D. (2012). Games and mathematics. Subtle connections. New York: Cambridge University Press.

Yıldırım, A., \& Şimşek, H. (2016). Sosyal bilimlerde nitel araştırma yöntemleri (10th ed.). Ankara: Seçkin.

Yin, R. K. (2011). Qualitative research from start to finish. New York, NY: Guilford Press.

Zaslavsky, C. (1998). Math games and activities from around the world. Chicago: Chicago Review Press.

Zaslavsky, C. (2003). More math games and activities from around the world. Chicago: Chicago Review Press.

\section{Notes}

Note 1. It was stated by my student that father of my student's grandfather learned this game from an Iranian and spread it to his relatives in the village. This person passed away at the age of 96 in the process of the 2020 pandemic. This suggests that the game has been played in the village for at least more than 100 years.

Note 2. I learned that the other person was 75 years old and had difficulty remembering some information.

\section{Copyrights}

Copyright for this article is retained by the author(s), with first publication rights granted to the journal.

This is an open-access article distributed under the terms and conditions of the Creative Commons Attribution license (http://creativecommons.org/licenses/by/4.0/). 